\title{
Calculation of Magnetic Hysteresis Minor Loop and New Features Extraction based on Magnetic Barkhausen Noise
}

\author{
Ping Wang ${ }^{1}$, Kai Huang ${ }^{2}$, Jianqin $\mathrm{Xu}^{2}$, Mengdi Li ${ }^{1}$, Zhibin Zhang ${ }^{2}$, Guiyun Tian ${ }^{1,3}$, Huamin Zhang ${ }^{2}$, Grzegorz Psuj ${ }^{4}$ \\ ${ }^{1}$ College of Automation Engineering, Nanjing University of Aeronautics and Astronautics, Nanjing 210016, P.R. China \\ ${ }^{2}$ Special equipment Safety Supervision Inspection Institute of Jiangsu Province, Nanjing 210036, P.R. China \\ ${ }^{3}$ School of Electrical and Electronic Engineering, Newcastle University, Newcastle NE1 7RU, UK \\ ${ }^{4}$ Faculty of Electrical Engineering, West Pomeranian University of Technology, PL-70313 Szczecin, Poland
}

\begin{abstract}
Magnetic Barkhausen Noise (MBN) is an effective non-destructive testing approach for ferromagnetic material residual stress measurement and evaluation. However, the common MBN signal features such as the time to peak value and frequency spectrum are not accurate enough for stress evaluation due to the randomness of MBN signal. This paper proposed a new method to calculate a curve which looks like magnetic hysteresis loop (B-H curve) based on the line integral from envelope curve of MBN signal. And some new features are extracted from the above integrated B-H curve. These new signal features show a good linear relationship with applied stress. By comparing these new features with previous common features, we find that the envelope area and center height difference of new features can effectively reduce the adverse impact on stress evaluation due to the randomness of MBN signal.
\end{abstract}

\section{Introduction}

The ferromagnetic materials, which is composed by many small magnetic domains with different orientations, can be magnetized by the external magnetic field as the orientations of magnetic domains turn to the direction of the external magnetic field. In the process of magnetization, a series of magnetic pulses (Magnetic Barkhausen Noise, MBN) and mechanical vibrations (Magnetic Acoustic emission, MAE) occurs with the movement of the boundaries and the changing of the directions of the magnetic domains. The movement of the magnetic domains is influenced by the micro-structure features as well as by the stress of the materials, so the information about these features and stress can be detected by the observation of the MBN and $\mathrm{MAE}^{[1][2]}$.

When the dependence of MBN on stress is analyzed, frequently used feature values are: power spectrum ${ }^{[3]}$, root mean square(RMS $)^{[4]}$, mean and ring numbers $(\mathrm{Nr})^{[5]}$. And the envelope of MBN signal is also extracted to obtain peak(Vp), peak time(Tp), full width at half maximum $(\mathrm{FWHM})^{[6]}$ and other information.Because of the randomness of MBN signal, difference between two measurements is obvious, when peak time or FWHM is used as feature value. And the ring numbers can be affected by threshold and intercept time.

\section{MBN signal and B-H curve}

There will be magnetization in the ferromagnetic material under the effect of external magnetic field. When the saturation occurs in the ferromagnetic materials, the magnetic state is not restored to its original state after removing the magnetic field. When the magnetic field changes back and forth in the positive and negative directions, the magnetization of the media is in a cycle, thus forming a hysteresis $\operatorname{loop}^{[7]}$ (see Fig. 1) . By observing the hysteresis loop of the ferromagnetic materials we find that the curve displays a step-jitter state in the irreversible magnetization phase, rather than a smooth and continuous curve. The coil placed on the surface of the specimen produces a noise pulse in the form of voltage indicating that the magnetization process of the ferromagnetic materials is not continuous ${ }^{[8]}$.

Some researchers found that the shapes of hysteresis loops, coercivity and remanence changed in some regularity with the difference applied stress. With the increase of tensile stress which is parallel to the direction of the external magnetic field, both of the coercivity and the remanence increase. While with the increase of compressive stress, both of the coercivity and the remanence decrease $\mathrm{e}^{[9]}$.

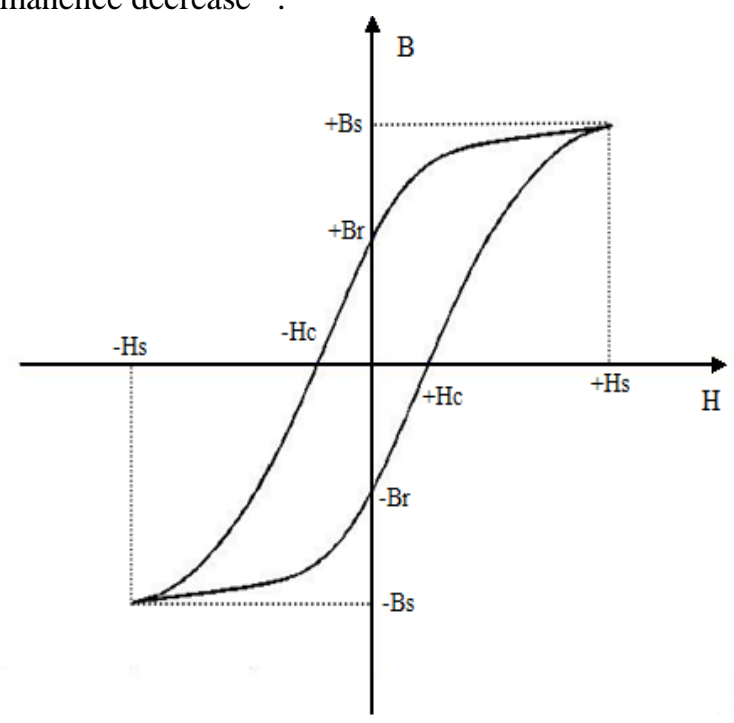

Fig.1. B-H curve

When we extracted the envelope of MBN signal, we found that the envelope trend is similar to the slope trend of B-H curve. We assume that: a) at any point, the amplitude of the MBN signal reflects the number of the domain movement of the ferromagnetic material. b) The number of the domain movement at this point results in a change of B in the magnetization process. c) Total number of the domain movement results in the accumulation of $B$. Based on these assumptions, we believe that we can get some information about B from the integration of the 
amplitude of the MBN signal. And we can also extract new features from integration curve to evaluate stress level.

\section{Hysteresis loop projection and features extraction}

Two methods can be considered to realize curve integration, one is integrate over time, the other is over amplitude of excitation signal. When time is used as independent variable, samples are at equal intervals and can be easily calculated. As the sinusoidal signal is used as excitation, if amplitude of excitation signal is used as independent variable, the excitation signal should be smoothed, and corresponded to the envelope of MBN signal. Since there is a phase shift between the excitation and the MBN signal after smoothing and filtering, it's hard to correspond them exactly. And the Calculation process will be very complicated. So we decided to integrate over time.

After the pretreatment of MBN signal, we set the peak of excitation signal as outset to intercept ten cycles of MBN signal. Then extract its envelope and seek 10 cycles average as the envelope to be integrated. We integrate the first half cycle of the envelope curve and then integrate the last half but with a shift of a certain value, which is determined by the integral value of the first half cycle.After that, the last half cycle integration curve is flipped to form a loop with the last half cycle integration curve. Fig. 2 shows the envelope curve before integration and the integration loop.The integration curve is unclosed because the MBN signal is asymmetric, which is caused by the remanence in specimen. To eliminate the influence of remanence in specimen, demagnetization of specimen is required before experiment.
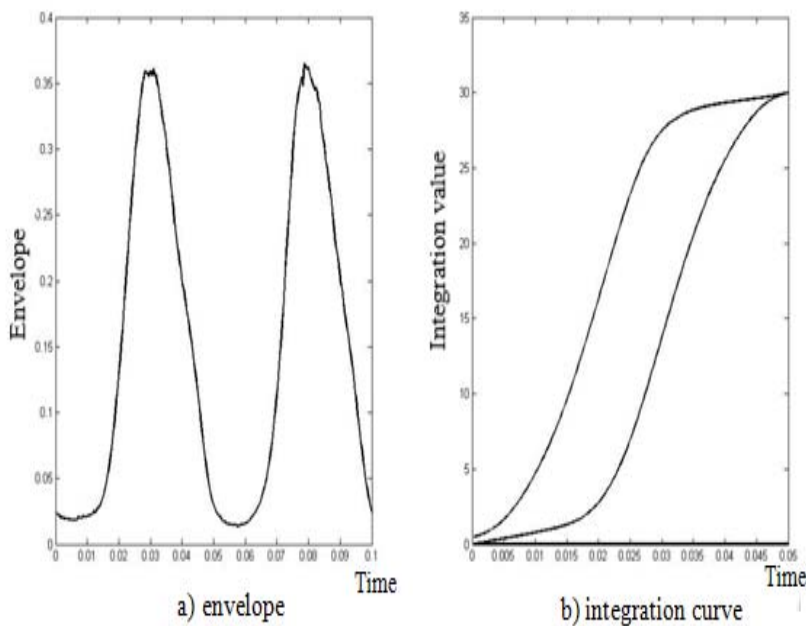

Fig.2. Envelope of MBN signal and its integration curve

Since B-H curve is obtained by integrating MBN signal envelope, we can get maps of envelope features from integration curve. Obviously, maximum slope of the curve is the peak of MBN signal, and the location of maximum slope is the peak time. Furthermore, we extracted new features as follows, which are based on the shape of the integration curve and the parameters of B-H curve.

a) Envelope Area(Ea): value of last point of integration curve (see Fig.3)

b) Center Width(Wc): time difference between first half curve and last half curve when the integration value reach $\mathrm{Ea} / 2$. And the center width of $\mathrm{B}-\mathrm{H}$ curve is twice of coercivity (Hc) . (see Fig.3)

c) Center Height Difference(Hdc): height difference between half curve and last half curve at Ts/4(Ts is cycle time) . And the center height difference of $\mathrm{B}-\mathrm{H}$ curve is twice of remanence(Br). (see Fig.3)

d) Maximum Width(Wm): maximum time difference between first half curve and last half curve (see Fig.3)

e) Maximum Height Difference(Hdm): maximum height difference between half curve and last half curve (see Fig.3)

f) Loop Area(La): area of integration loop (see Fig.3)
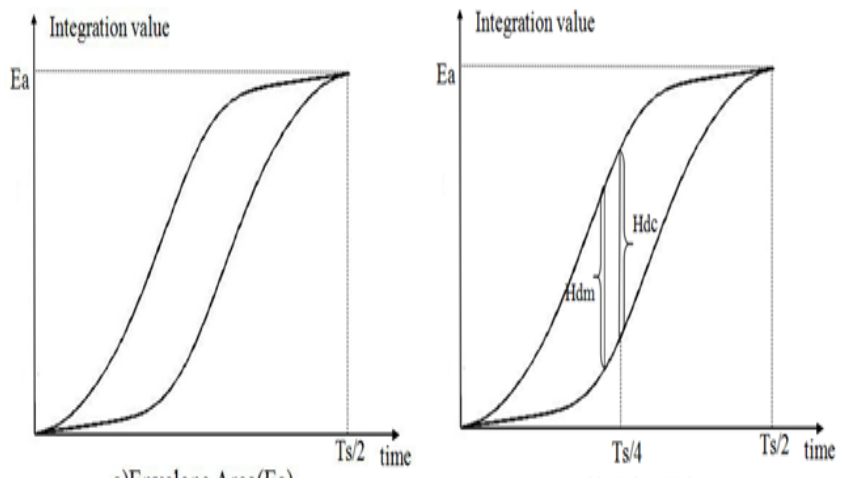

b) Hdc\&Hdm
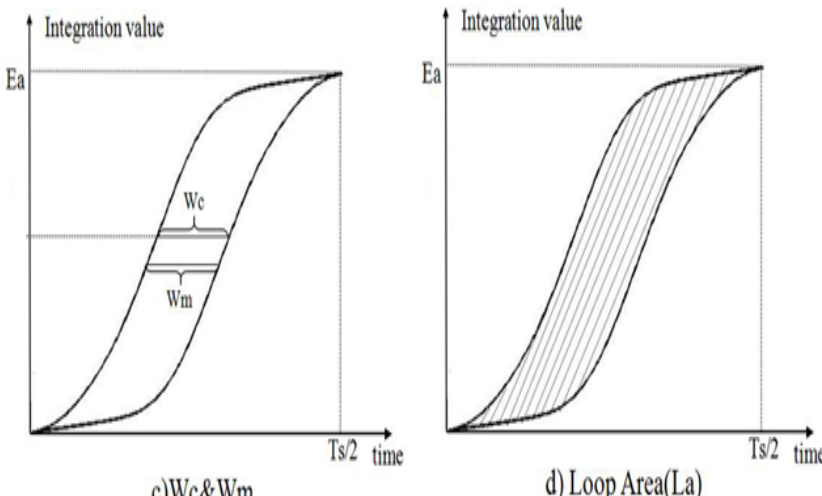

c)Wc\&Wm

Fig.3. New features

\section{Experimental verification}

The experimental platform is composed with a four-point bending load platform(see Fig.4) and our stress detection system which is based on Barkhausen effect(see Fig.5). We use A3 steel block as load specimen, whose size is $660 \mathrm{~mm} \times 30 \mathrm{~mm} \times 10 \mathrm{~mm}$. In order to test both the tensile and compressive stress of the specimen, the MBN probe is placed on the middle surface of both the top and the bottom of the specimen. And we use equation 1 to calculation stress in the position of the probe. F means the force we load on the specimen, c means the distance between point $\mathrm{A}$ and point $\mathrm{B}$ (the distance between point $A^{\prime}$ and point $B^{\prime}$ is also c). $b$ means the width of specimen and $h$ is the thickness of specimen. When the force loaded 
on the specimen range from 0 to $800 \mathrm{~N}$, the stress change from 0 to $120 \mathrm{MPa}$.The frequency of sinusoidal excitation applied on the drive coil is $10 \mathrm{~Hz}$ with different amplitude of $1.6 \mathrm{~V}, 2.1 \mathrm{~V}$ and $2.6 \mathrm{~V}$.

$$
\sigma=E \varepsilon=\frac{3 F C}{b h^{2}}
$$

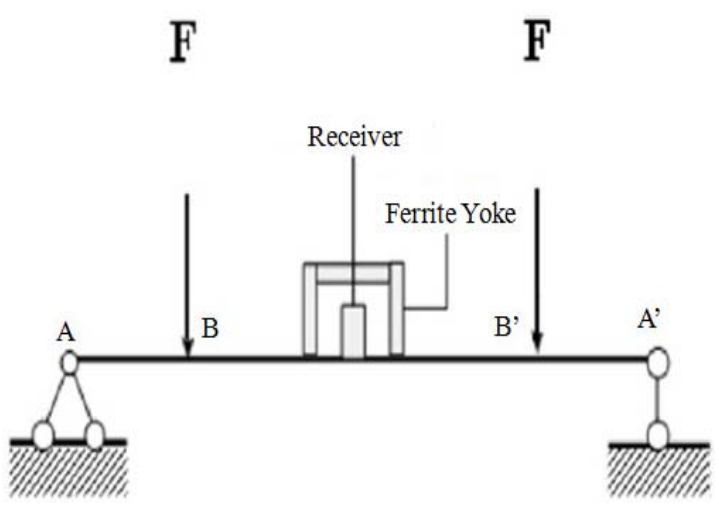

Fig.4. Stress loading model diagram

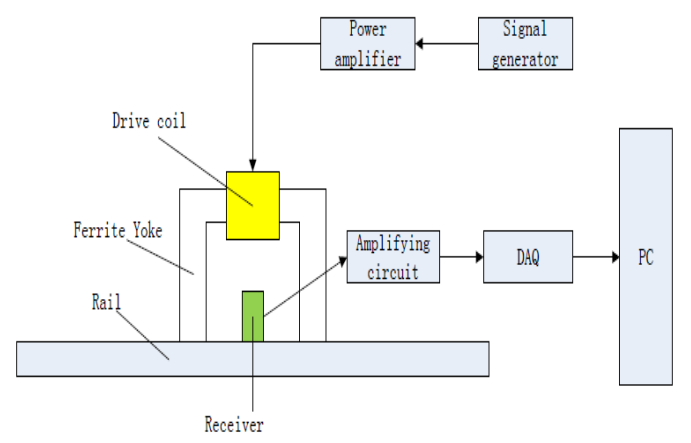

Figure.5.Measurement system structure diagram

\subsection{New features extraction}

The parameters illustrated before were used in the experiment. Then we normalized data to see how the features change when the stress changed(see Fig.6). The negative stress means compressive stress and the positive stress is tensile. As the figure illustrate, each feature increase with the increase of tensile stress or with the decrease of compressive stress under 120MPa.And there is a linear relationship between envelope area and stress. Besides, Wc and Hdc show the same variation trend as Hc and $\mathrm{Br}$ when the stress change.
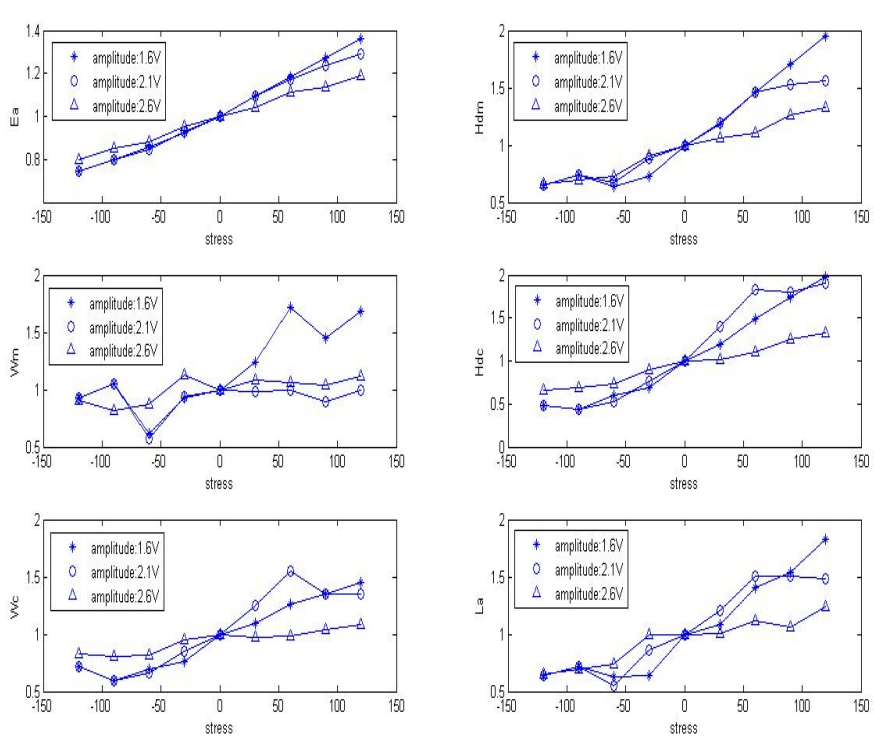

Fig.6. Features and stress

\subsection{Features comparison}

It is known that the volatility of feature value which caused by randomness of MBN signal is the obstacle when evaluate stress via MBN signal. In order to evaluate the effect of volatility of feature value on certainty of stress evaluation, we integrated the envelope every single cycle and extracted mean and standard deviation of features in each cycle. And we also obtained the variation and maximum standard deviation of mean when stress changes from $0 \mathrm{MPa}$ to $120 \mathrm{MPa}$. Variation of mean illustrate variation range of features when stress changes while maximum standard deviation of mean shows certainty of data we get. The ratio of delta mean and maximum standard deviation of mean can used as a weigh of the effect of volatility of feature value, when we use it to evaluate the magnitude of stress.

Tab. 1 shows the result of above-mentioned method with excitation amplitude of $1.6 \mathrm{~V}$ loaded with compressive stress. Tab. 2 shows the result loaded with tensile stress. The table illustrate that, Ea, Hdc and Hdm show minor volatility than others in new features.They have better capability to decrease the uncertainty which caused by randomness of MBN signal and evaluate stress much more accurate than peak time, FWHM and ring numbers.

\section{Conclusions}

In this paper, the relationship between Barkhausen noise and hysteresis loop is analyzed, and some hypothesis are proposed for the calculation of magnetic hysteresis 'minor' Loop. we proposes and investigates a new method to calculate this curve by the integration of envelope of MBN signal and some new features are extracted from the above integrated B-H curve and concluded as follows: 
a) It is possible to calculate magnetic hysteresis loop by intercept, average and integrate the envelope of MBN signal.

b) The remanence in specimen cause the asymmetric of MBN signal and misclosure of integration curve. To eliminate the influence of remanence in specimen, demagnetization of specimen is required before experiment.

c) The features extracted from integration curve show monotonic trend with stress significantly: each feature increase with the increase of tensile stress or with the decrease of compressive stress under $120 \mathrm{MPa}$.And there is a linear relationship between envelope area and stress.

d) Wc and Hdc show the same variation trend as Hc and $\mathrm{Br}$ when the stress change. With the increase of tensile stress, they all increase.While with the increase of compressive stress, they all decrease.

e)When the ratio of delta mean and maximum standard deviation of mean is uesd as a weigh of the effect of volatility of feature value, Ea, Hdc and Hdm have statistical properties to improve the uncertainty which caused by randomness of noise, when it is used to evaluate the magnitude of stress.

Tab.1. Comparison of Features with compressive stress loaded

\begin{tabular}{ccccccccccccc}
\hline Features & Ea & Hdm & Wm & Hdc & Wc & La & Vp & Tp & FWHM & RMS & Mean & Nr \\
\hline Max Std & 0.617 & 0.471 & 37.296 & 0.515 & 3.838 & 189.024 & 0.009 & 399.56 & 336.66 & 0.289 & 0.123 & 14.157 \\
Delta Mean & 5.925 & 2.35 & 30.646 & 2.54 & 11.434 & 492.11 & 0.042 & 125 & 240 & 2.772 & 1.354 & 18.7 \\
$\quad$ ratio & 0.104 & 0.2 & 1.217 & 0.203 & 0.336 & 0.334 & 0.221 & 3.196 & 1.403 & 0.104 & 0.091 & 0.757
\end{tabular}

Tab.2. Comparison of Features with tensile stress loaded

\begin{tabular}{ccccccccccccc}
\hline Features & Ea & Hdm & Wm & Hdc & Wc & La & Vp & Tp & FWHM & RMS & Mean & Nr \\
\hline Max Std & 0.903 & 0.958 & 41.775 & 0.902 & 4.337 & 302.08 & 0.012 & 459.14 & 332.12 & 0.351 & 0.145 & 19.622 \\
Delta Mean & 8.712 & 4.784 & 38.012 & 4.831 & 12.953 & 647.26 & 0.07 & 592 & 240 & 4.354 & 2.109 & 18.7 \\
ratio & 0.104 & 0.2 & 1.099 & 0.187 & 0.335 & 0.467 & 0.166 & 0.776 & 1.333 & 0.081 & 0.069 & 1.055 \\
\hline
\end{tabular}

\section{Acknowledgments}

The research of this paper is supported by FP7 Health monitoring of offshore wind farms (HEMOW) project, National Science Foundation of China (50907032/E070104), "Research and equipment development of lifting machinery structure fatigue and stress distribution rapid detection ", science and technology project of General Administration of Quality Supervision, Inspection and Quarantine of the People's Republic of China(2013OK14)

corresponding author: Kai Huang

\section{References}

[1] Kevin S. Opoku, Determining the onset of grinding burn using magnetic Barkhausen noise. Academic Year 2004-2005

[2] P. Wang, Y. Gao, Y. Yang, G.Y. Tian, E. Yao, H. Wang, Experimental Studies and New Feature Extractions of MBN for Stress Measurement on Rail Tracks. IEEE Transactions on Magnetics. 49(8): 4858-4864, 2013.

[3] Hechi Y. Application of Magnetic Barkhausen Noise Technique for Residual Stress Measuring to the Evaluation of Heat Treatment Process [J]. Heat Treatment, 2008, 28(2):17-20.

[4] Wang $\mathrm{P}$, Ji $\mathrm{X}$, Yan $\mathrm{X}$, et al. Investigation of temperature effect of stress detection based on Barkhausen noise[J]. Sensors and Actuators A: Physical, 2013.
[5] Ping WANG, Shougao ZHU, Guiyun TIAN, Haitao WANG, John Wilson, Xin WANG, Stress Measurement Using Magnetic Barkhausen Noise and Metal Magnetic Memory Testing, Meas. Sci. Technol. 21 (2010), pp. 1-6.

[6] Yarong Y. Research on Magnetic Barkhausen Noise for Railtrack Stress Detection[D]. Nanjing:Nanjing University of Aeronautics and Astronautics, 2011.

[7] Roxana Hutanu, A correlation study between magnetic Barkhausen noise and intergranular residual stresses in steels, [Ph.D. thesis of Queen's University], Kingston, Queen's University, October, 2005.

[8] Qiujun Z. Development and Research of the Equipment of Barkhausen Noise Rail Stress Detection[D]. Nanjing:Nanjing University of Aeronautics and Astronautics, 2012.

[9] Bing Z. Study of micromagnetics of ferromagnetic film cell under stress[D]. Chengdu: University of Electronic Science and Technology of China, 2008. 